EPJ Web of Conferences 66, 04020 (2014)

DOI: $10.1051 /$ epjconf/ 20146604020

(C) Owned by the authors, published by EDP Sciences, 2014

\title{
Detecting the anti-hypertriton and anti-helium-4 from the RHIC
}

\author{
Yu-Gang $\mathrm{Ma}^{1, \mathrm{a}}$ \\ ${ }^{1}$ Shanghai Institute of Applied Physics, Chinese Academy of Sciences, Shanghai 201800, China
}

\begin{abstract}
Recent progress on the dectection of antimatter particles at RHIC is briefly reviewed. The observations of the anti-hypertriton $\left({ }_{\bar{\Lambda}}^{3} \overline{\mathrm{H}}\right)$ and anti-helium-4 nuclei $\left({ }^{4} \overline{\mathrm{He}}\right.$, or $\bar{\alpha}$ ) from the RHIC-STAR Collaboration are highlighted. In addition, preliminary lifetime measurement of ${ }_{\Lambda}^{3} \mathrm{H}$ and energy dependence of strangeness population factor are presented. The mechanism of light antinuclei production is also discussed.
\end{abstract}

\section{Introduction}

Relativistic heavy-ion collisions create suitable conditions for a phase transition from hadron to deconfined quark matter which was predicted by the Lattice QCD calculation. During the collision, a hot and dense partonic matter, i.e. so-called Quark-Gluon Plasma (QGP), can be formed. Many evidences have demonstrated that a strong coupled QGP matter has been produced in central $\mathrm{Au}+\mathrm{Au}$ collisions at RHIC energies [1]. Different from elementary particle collisions, large amounts of energy are deposited into a more extended volume than nucleon size. These nuclear interactions produce hot and dense matter containing roughly equal numbers of quarks and antiquarks. Then the QGP expands rapidly and cools down and undergoes a transition into a hadron gas, producing, among others, nucleons and their antiparticles. Therefore the relativistic heavy-ion collision can not only provide an environment to study strong interacting phase transition and properties of QCD matter but also an ideal venue to produce antimatter particles.

The ideal of antimatter can be traced back to the end of 1890s, when Schuster discussed a hypothesis of the existence of antiatoms as well as antimatter solar system by hypothesis in his letter to Nature magazine [2]. However, the modern concept of antimatter is originated from the negative energy state solution of a quantum-mechanical equation, which was proposed by Dirac in 1928 [3]. Two years later, C. Y. Chao found that the absorption coefficient of hard $\gamma$-rays in heavy elements was much larger than that was expected from the Klein-Nishima formula or any other [4]. This "abnormal" absorption is in fact due to the creation of the pair of electron and its anti-partner, so-called positron. This experiment gives the first indirect observation of the first anti-matter particle, namely positron. Two years later, Anderson observed positrons by a cloud chamber [5]. Since the observation of the anti-proton $(\overline{\mathrm{p}})$ in 1955 , antimatter nuclei such as $\overline{\mathrm{d}},{ }^{3} \overline{\mathrm{H}},{ }^{3} \overline{\mathrm{He}}$ have been widely studied in both cosmic rays and accelerator experiments for the purposes of dark matter exploration and the study of manmade matter such as quark gluon plasma, respectively [6] .

The recent progress regards the observation of antihypertriton $\left({ }_{\bar{\Lambda}}^{3} \overline{\mathrm{H}}\right)$ [7] and antihelium-4 $\left({ }^{4} \overline{\mathrm{He}}\right.$, or $\bar{\alpha}$ ) [8] nucleus in Relativistic Heavy Ion Collider (RHIC) reported by the STAR experiment as well

\footnotetext{
ae-mail: ygma@sinap.ac.cn
} 
as the longtime confinement of antihydrogen atoms [9] based on an antiproton decelerator facility by ALPHA collaboration have already created a lot of excitation in both nuclear and particle physics community. All of the measurements performed above have implications beyond the fields of their own. For instance, the study of hypernucleus in heavy ion collisions is essential for the understanding of the interaction between nucleon and hyperon (YN interaction), which plays an important role in the explanation of the structure of neutron stars. Furthermore, as we learned from heavy ion collisions, the production rate for ${ }^{4} \overline{\mathrm{He}}$ produced by colliding the high energy cosmic rays with interstellar materials is too low to be observed. Even one ${ }^{4} \overline{\mathrm{He}}$ or heavier antinucleus observed in the cosmic rays should be a great hint of the existence of massive antimatter in the Universe. Furthermore, the successful trap of antihydrogen atoms can lead to a precise test of the CPT symmetry law, as well as a measurement of the gravitational effects between antimatter and matter in the future.

In this paper, we focus on the above mentioned discoveries on antihypertriton [7] and antihelium-4 [8] at the RHIC as well as the understanding for these anti-nuclei production at heavy-ion collisions. A brief review on the formation and observation of ${ }_{\bar{\Lambda}}^{3} \overline{\mathrm{H}}$ through the secondary vertex reconstruction via decay channel ${ }_{\bar{\Lambda}}^{3} \overline{\mathrm{H}} \rightarrow{ }^{3} \overline{\mathrm{He}}+\pi^{+}$with a branching ratio of $25 \%$ in high energy heavy ion collisions is presented in Sec. 2. Also the related hypernuclear physics is mentioned. Section 3 discusses the particle identification of ${ }^{4} \overline{\mathrm{He}}$ nucleus by energy loss in Time-Projection-Chamber (TPC) as well as by measuring their mass value directly by Time Of Flight (TOF) at RHIC-STAR. The antimatter nuclei production mechanism is briefly described in Section 4. Finally we give a summary in Section 5.

\section{The first antimatter hypernucleus: ${ }_{\bar{\Lambda}}^{3} \overline{\mathrm{H}}$}

Different from the normal (anti-)nuclei which only consist of (anti-) up (u) and down (d) quarks, (anti-)hypernucleus also includes the (anti-)strange quark degree of freedom, of which the typical one is $\Lambda$-hypernucleus. The simplest hypernucleus observed so far is hypertriton, which is composed of one neutron, one proton and one $\Lambda$-hyperon. Due to the presence of hyperon, hypernucleus provides an ideal environment to learn the hyperon-nucleon interaction, which is responsible partly for the binding of hypernuclei and lifetime. So far, many hypernuclei have been identified, even for the observation of double- $\Lambda$ hypernucleus. However, no anti-hypernucleus was observed until the STAR Collaboration announced the observation of first anti-matter hypernucleus, i.e. ${ }_{\Lambda}^{3} \overline{\mathrm{H}}$ [7], in 2010. In the technique viewpoint, the identification of $\frac{3}{\bar{\Lambda}} \overline{\mathrm{H}}$ can be achieved by reconstructing their secondary vertex via the decay channel of ${ }_{\bar{\Lambda}}^{3} \overline{\mathrm{H}} \rightarrow{ }^{3} \overline{\mathrm{He}}+\pi^{+}$, which occurs with a branching ratio of $25 \%$ (assuming that this branching fraction is the same as that for ${ }_{\Lambda}^{3} \mathrm{H}$ [10]) [7, 11]. The data used for ${ }_{\bar{\Lambda}}^{3} \overline{\mathrm{H}}$ analysis was collected by the STAR experiment at RHIC, using the cylindrical TPC, which is 4 meters in diameter and 4.2 meters long in the beamline direction. The identification of tracks can be achieved by correlating their ionization energy loss $\langle d E / d x\rangle$ in TPC with their magnetic rigidity.

Topological cuts including the distance between two daughter tracks ${ }^{3} \overline{\mathrm{He}}$ and $\pi^{+}(<1 \mathrm{~cm})$, distance of closest approach (DCA) between ${ }_{\bar{\Lambda}}^{3} \overline{\mathrm{H}}$ and primary vertex $(<1 \mathrm{~cm})$, decay length of ${ }_{\bar{\Lambda}}^{3} \overline{\mathrm{H}}(>2.4 \mathrm{~cm})$, and the DCA of $\pi$ track $(>0.8 \mathrm{~cm})$, are employed to enhance the signal to background ratio. The invariant mass of ${ }_{\Lambda}^{3} \mathrm{H}$ and ${ }_{\bar{\Lambda}}^{3} \overline{\mathrm{H}}$ were calculated based on the conservation of momentum and energy in the decay process. The results are shown in Figure 1A for ${ }_{\Lambda}^{3} \mathrm{H}$ and Figure $1 \mathrm{~B}$ for ${ }_{\bar{\Lambda}}^{3} \overline{\mathrm{H}}$. The successfully reproduced combinatorial background with a rotation strategy can be described by double exponential function: $f(x) \propto \exp \left[-\left(x / p_{1}\right)\right]-\exp \left[-\left(x / p_{2}\right)\right]$, where $x=m-m\left({ }^{3} H e\right)-m(\pi)$, and $p_{1}, p_{2}$ are the free parameters. Finally, the signals are counted by subtracting the double exponential background of ${ }_{\Lambda}^{3} \mathrm{H}$ and ${ }_{\bar{\Lambda}}^{3} \overline{\mathrm{H}}$. 

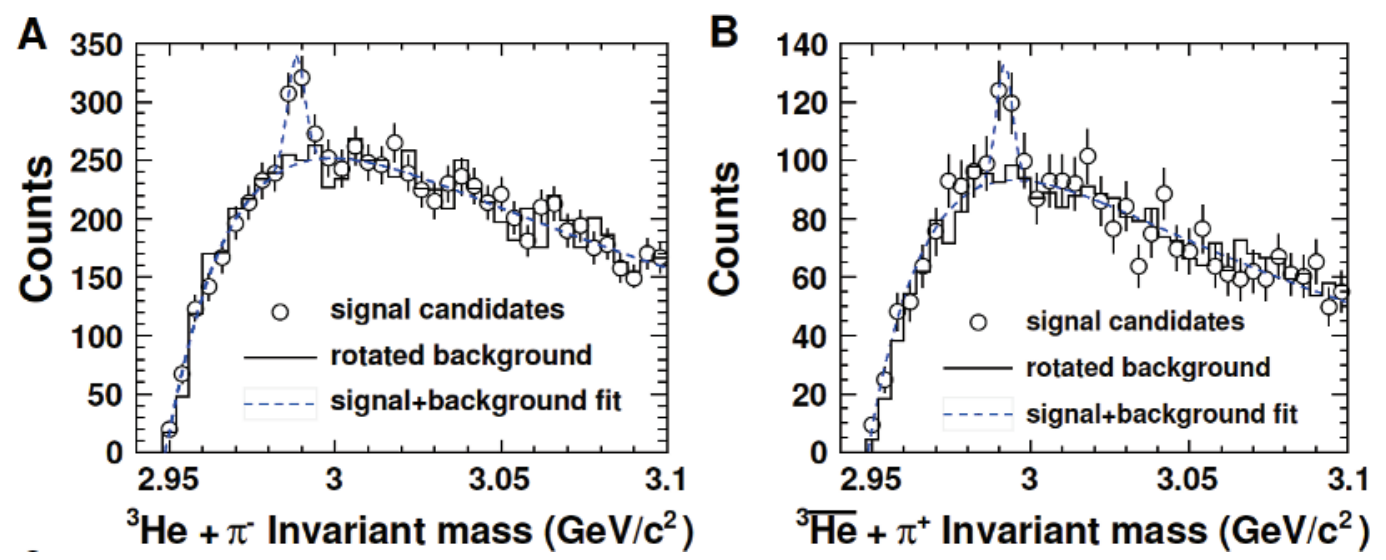

Figure 1. (A and B) Reconstructed invariant mass distribution of ${ }^{3} \mathrm{He}$ and $\pi$ for ${ }_{\Lambda}^{3} \mathrm{H}$ and ${ }_{\bar{\Lambda}}^{3} \overline{\mathrm{H}}$, respectively. Open circles stand for the signal distribution, while solid lines are the rotated combinatorial background. Blue dashed lines are the Gaussian (signal) plus double exponential (background) function fit to the distribution. Adapted from the Ref. [7].
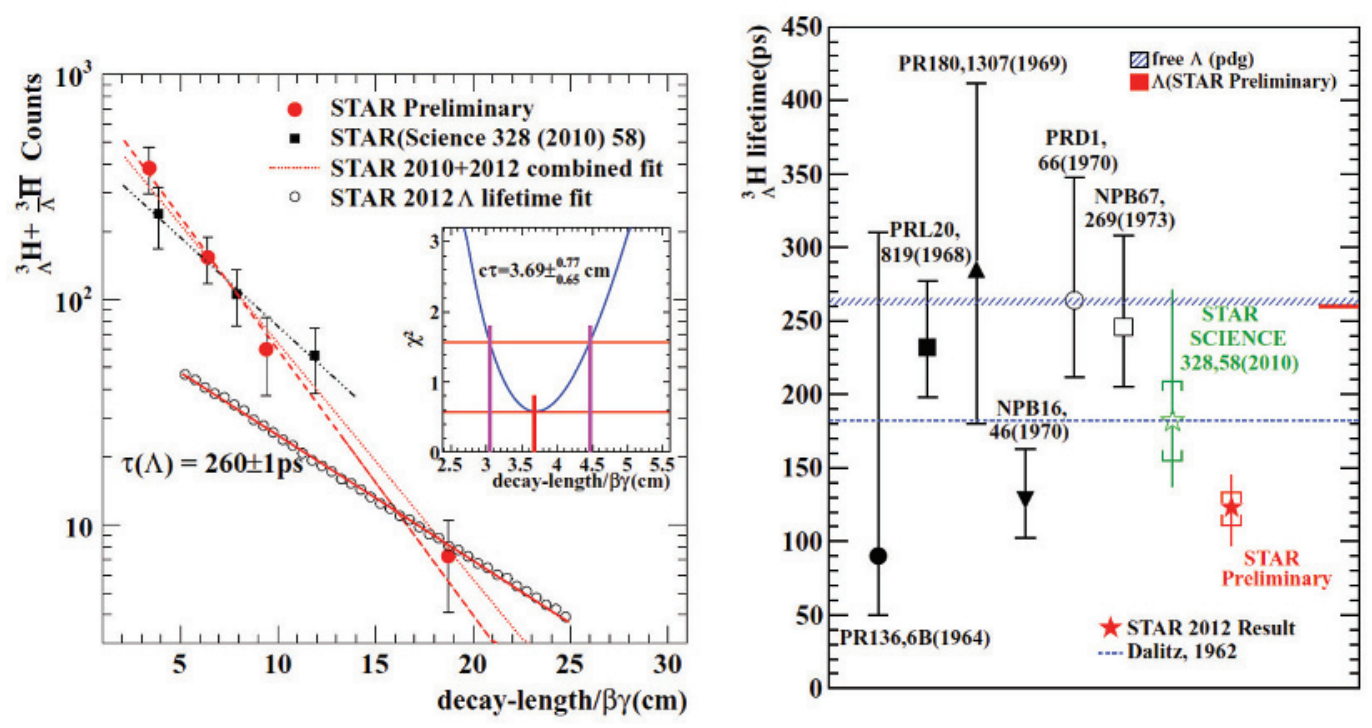

Figure 2. (Left) The yields of ${ }_{\bar{\Lambda}}^{3} \overline{\mathrm{H}}$ (solid squares) and $\Lambda$ (open circles) vs $c \tau$ distribution. The solid lines stand for the $c \tau$ fits, and the insert plot describes $\chi^{2}$ distribution of the best fits. (Right) Comparison between the present measurement and theoretical calculation [10,12], as well as the previous measurements. Adapted from the Ref. [13].

The measurement of ${ }_{\Lambda}^{3} \mathrm{H}\left({ }_{\bar{\Lambda}}^{3} \overline{\mathrm{H}}\right)$ lifetime provides us an effective tool to understand the $\mathrm{Y}(\Lambda)-\mathrm{N}(\mathrm{p}, \mathrm{n})$ interactions $[10,12]$. And, the secondary vertex reconstruction of ${ }_{\Lambda}^{3} \mathrm{H}\left({ }_{\bar{\Lambda}}^{3} \overline{\mathrm{H}}\right)$ allow us to perform a calculation of its lifetime, via equation $N(t)=N(0) \exp (-t / \tau)$, where $t=l /(\beta \gamma c), \beta \gamma c=p / m, l$ is the decay length of ${ }_{\Lambda}^{3} \mathrm{H}, p$ is their momentum, $m$ is their mass value, while $c$ is the speed of light. ${ }_{\Lambda}^{3} \mathrm{H}$ and 

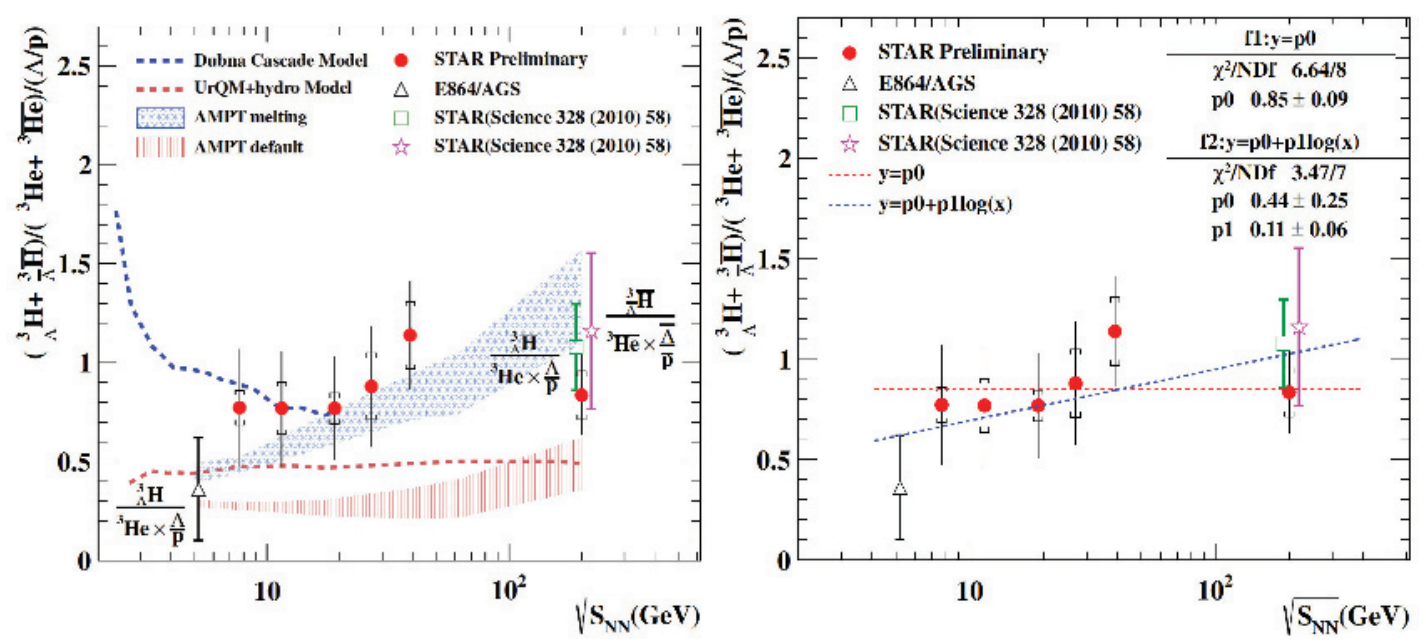

Figure 3. (Left) Beam energy dependence of $S_{3}$. Lines and shadows: model calculation results [16, 17]. Markers: experimental results. (Right) Quantitative fit of the data points. Adapted from the Ref. [13].

${ }_{\bar{\Lambda}}^{3} \overline{\mathrm{H}}$ samples are combined together to get a better statistics, with the assumption of the same lifetime of ${ }_{\Lambda}^{3} \mathrm{H}$ and ${ }_{\bar{\Lambda}}^{3} \overline{\mathrm{H}}$ base on the CPT symmetry theory. The measured yield is corrected for the tracking efficiency and acceptance of TPC, as well as the reconstruction efficiency of ${ }_{\Lambda}^{3} \mathrm{H}$ and ${ }_{\bar{\Lambda}}^{3} \overline{\mathrm{H}}$. Then, the $l /(\beta \gamma)$ distribution can be fitted with an exponential function to extract the lifetime parameter $c \tau$.

The RHIC beam energy scan program in 2010-2011 allowed STAR to collect data for $\mathrm{Au}+\mathrm{Au}$ collisions over a broad range of energies. To get an even better statistics, datasets are combined in the lifetime measurement. The best fitting with $\chi^{2}$ minimization method yielded a lifetime of $123 \pm{ }_{22}^{26}$ (stat) \pm 10 (sys) ps, as shown in left panel of Figure 2. Right panel of Figure 2 shows a comparison of the present measurement with theoretical calculation [10,12], as well as the previous measurements [13]. As a comparison, STAR $2010{ }_{\Lambda}^{3} \mathrm{H}$ lifetime measurement [7] and the STAR $2010+2012$ combined results are also provided. The current measurement is consistent with the STAR 2010 measurement within $1.5 \sigma$ and is statistically improved. In this lifetime measurement, two kinds of sources for systematic study are considered: 1 . choice of V0 topology cuts; 2 . choice of bin width and invariant mass range. These effects contribute to the final systematic error. Additional sources of loss, like the interaction between ${ }_{\Lambda}^{3} \mathrm{H}$ and material (air+detector) are also considered, which can be neglected due to its less than $1.5 \%$ effect. As a further cross-check, $\Lambda$ is reconstructed via the $\Lambda \rightarrow p+\pi^{-}$decay channel. We use exactly the same method to obtain the $\Lambda$ lifetime and the result is $260 \pm 1$ ps which is consistent with the $\tau=263 \pm 2$ ps compiled by the Particle Data Group [14]

In hot and dense environment, high production rate of ${ }_{\Lambda}^{3} \mathrm{H}\left({ }_{\bar{\Lambda}}^{3} \overline{\mathrm{H}}\right)$ due to equilibration among strange quarks and light quarks $(\mathrm{u}, \mathrm{d})$ is proposed to be a signature of the formation of QGP $[7,15]$. By comparing the yields of ${ }_{\Lambda}^{3} \mathrm{H}$ and ${ }^{3} \mathrm{He}$, the baryon strangeness correlation factor can be extracted. Our recent calculation [16] indicates that the strangeness population factor, $S_{3}={ }_{\Lambda}^{3} \mathrm{H} /\left({ }^{3} \mathrm{He} \times \Lambda / p\right)$ is an effective tool to distinguish QGP phase and pure hadronic phase. The definition of $S_{3}$ incorporates the $\Lambda / \mathrm{p}$ ratio in order to remove the sensitivity on yield differences on $\Lambda$ and $\mathrm{p}$ as a function of the beam energy. It is interesting to note that $S_{3}$ increases with beam energy in a system with partonic interactions (Melting AMPT) while it is almost unchanged in a purely hadronic system (Default AMPT) as shown in the left panel of Fig. 3. The another model calculation based on UrQMD and Cascade 
model [17] is also plotted in Fig. 3. ${ }_{\Lambda}^{3} \mathrm{H} /{ }^{3} \mathrm{He}$ result for $\mathrm{Au}+\mathrm{Au}$ collisions at $200 \mathrm{GeV}$, in combination with the measured $\Lambda / p$ ratio from the same experiment allows us to deduce the measured $S_{3}$ at RHIC. From the trend of data points, it is hard to draw a conclusion directly. Therefore, a quantitative calculation is done by applying a zero-order and first-order fit to the data points, as shown in the right panel of Fig. 3. From the fit results, we can say that $S_{3}$ increases with increasing beam energy with $1.7 \sigma$ significance. The data imply that the local correlation strength between baryon number and strangeness is sensitive to the effective number of degrees of freedom of the system created at RHIC, and this number is significantly larger in a system dominated by partonic interactions compared with a pure hadronic gas.

\section{The heaviest antimatter nucleus observed so far: ${ }^{4} \overline{\mathrm{He}}$}

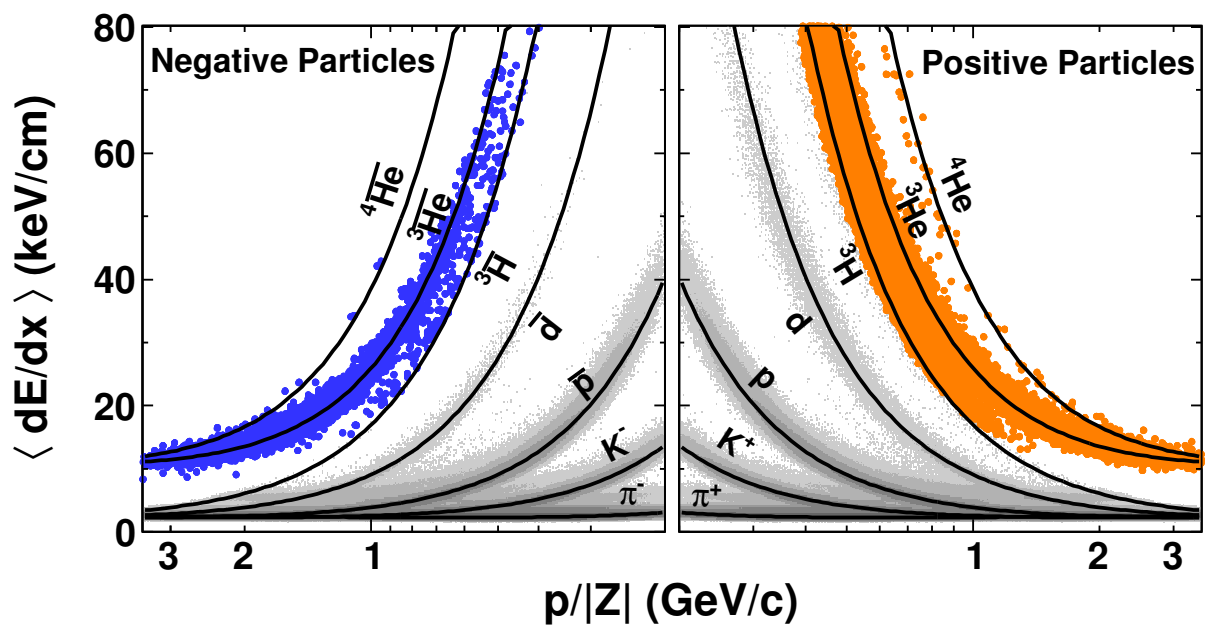

Figure 4. $\langle d E / d x\rangle$ as a function of $p /|Z|$ for negatively charged particles (left panel) and positively charged particles (right panel). The black curves represent the expected values for each particle species. The lower edges of the colored bands correspond to the HLT's online calculation of $3 \sigma$ below the $\langle d E / d x\rangle$ band center for ${ }^{3} \mathrm{He}$. The grey bands indicate the $\langle d E / d x\rangle$ of deuteron, proton, kaon, pion from Minimum Bias events at $200 \mathrm{GeV}$. Adapted from the Ref. $[8,18]$.

The STAR collaboration also made a first observation for ${ }^{4} \overline{\mathrm{He}}$ nucleus $[8,18]$ in April 2011 , with $10^{9} \mathrm{Au}-\mathrm{Au}$ collisions taken in the year 2007 and 2010. In additional to the particle identification method by combining energy loss $(\langle d E / d x\rangle)$ and rigidity provided by TPC, the observation of ${ }^{4} \overline{\mathrm{He}}$ nucleus relies on the measured traveling time of tracks given by the barrel TOF of the STAR experiment (Solenoidal Tracker At RHIC), which is composed of 120 trays, surrounding the TPC. The TPC is the central detector used in our measurements of antimatter which is situated in a solenoidal magnetic field and is used for three-dimensional imaging of the ionization trail left along the path of charged particles. Fig. 4 presents the $\langle d E / d x\rangle$ versus rigidity $(p /|Z|)$ distribution. The colored bands stand for the helium sample collected by High Level Trigger (HLT). A cut of the DCA less than $3 \mathrm{~cm}$ for negative tracks $(0.5 \mathrm{~cm}$ for positive tracks $)$ is used to reject the background. In the left panel, a couple of ${ }^{4} \overline{\mathrm{He}}$ candidates are identified and well separated from ${ }^{3} \overline{\mathrm{He}}$ at the low momentum region. A clear ${ }^{4} \mathrm{He}$ signal has been observed and centered around the expected $\langle d E / d x\rangle$ value of ${ }^{4} \mathrm{He}$ in the right 
panel. Considering that ${ }^{4} \overline{\mathrm{He}}$ candidates are mixed with ${ }^{3} \overline{\mathrm{He}}$ in high moementum region, we need to use the barrel TOF to assign the mass for He. With the barrel TOF, the mass value of particles can be calculated by the time of flight and path length of the track. With this TOF, $16{ }^{4} \overline{\mathrm{He}}$ candidates are identified. Together with $2{ }^{4} \overline{\mathrm{He}}$ candidates detected by TPC alone in the year 2007 which is presented in Fig.4, $18{ }^{4} \overline{\mathrm{He}}$ candidates are observed by the STAR experiment. We would mention that in this searching for ${ }^{4} \overline{\mathrm{He}}$, the online HLT was also very crucial since it was employed to select collisions which contain tracks with charge $Z e= \pm 2 e$ for fast analysis. The trigger efficiency for ${ }^{4} \overline{\mathrm{He}}$ is about $70 \%$ with respect to offline reconstruction, with a selection rate less than $0.4 \%$. So far, ${ }^{4} \overline{\mathrm{He}}$ is the heaviest antimatter nucleus observed in the world. Right after the public report of ${ }^{4} \overline{\mathrm{He}}$ from the STAR collaboration, the LHC-ALICE collaboration also observed some ${ }^{4} \overline{\mathrm{He}}$ particle candidates [19].

\section{Understanding production mechanism of antimatter light-nucleus}

Antimatter particles including $\overline{\mathrm{e}}, \overline{\mathrm{p}}, \overline{\mathrm{d}},{ }^{3} \overline{\mathrm{He}},{ }_{\bar{\Lambda}}^{3} \overline{\mathrm{H}},{ }^{4} \overline{\mathrm{He}}$ and antihydrogen atoms have been observed in the past eighty years. Most of these antimatter particles were produced by nucleon-nucleon reactions, where their production rate can be described by both thermodynamic model and coalescence model $[20,21]$. In a thermodynamic model, the system is characterized by the chemical freeze-out temperature $\left(T_{c h}\right)$, kinetic freeze-out temperature $\left(T_{k i n}\right)$, as well as the baryon and strangeness chemical potential $\mu_{B}$ and $\mu_{S}$, respectively. (Anti)nucleus is regarded as an object with energy $E_{A}=A m_{p}$ ( $A$ is the atomic mass number, $m_{p}$ is the mass of the proton) emitted by the fireball [20]. The production rate are proportional to the Boltzmann factor $e^{-m_{p} A / T}$ as shown in Equ. (1),

$$
E_{A} \frac{d^{3} N_{A}}{d^{3} P_{A}}=\frac{g V}{(2 \pi)^{3}} E_{A} e^{-m_{p} A / T} .
$$

where $P_{A}$ and $g$ are the momentum and degeneracy of (anti)nucleus, $V$ is the volume of the fireball.

On the other hand, in a coalescence picture, (anti)nucleus is formed by coalescence at the last stage of the system evolution since there exists strong correlation between the constituent nucleons in their phase space [22]. The production probability is described by Equ. (2),

$$
E_{A} \frac{d^{3} N_{A}}{d^{3} P_{A}}=B_{A}\left(E_{p} \frac{d^{3} N_{p}}{d^{3} P_{p}}\right)^{Z}\left(E_{n} \frac{d^{3} N_{n}}{d^{3} P_{n}}\right)^{A-Z},
$$

where $E \frac{d^{3} N}{d^{3} p}$ stands for the invariant yield of nucleons or (anti)nucleus, $Z$ is the atomic number, $p_{A}, p_{p}, p_{n}$ are the momentum of (anti)nucleus, protons and neutrons, when $p_{A}=A \times p_{p}$ is assumed. $B_{A}$ is the coalescence parameter.

Our calculation [21] based on the hydrodynamic motivated BlastWave model can reproduce the differential yields of $p(\overline{\mathrm{p}}), \Lambda(\bar{\Lambda})$, and light (anti)nuclei as well as (anti)hypertriton versus transverse momentum. Within the same framework, we make predictions for the production rates of ${ }_{\Lambda}^{3} \mathrm{H}\left({ }_{\bar{\Lambda}}^{3} \overline{\mathrm{H}}\right)$ and ${ }^{4} \mathrm{He}\left({ }^{4} \overline{\mathrm{He}}\right)$ etc by coupling with a naive coalescence model [21]. With those production rates, we can explore relative particle production abundance of (anti)nucleus and compare with data taken at RHIC. In a microscopic picture, both coalescence and thermal production of (anti)nucleus predict an exponential trend for the production rate as a function of baryon number. The exponential behavior of (anti)nucleus production rate in nuclear nuclear reaction has been manifested in Figure 5, which depicts the invariant yields $\left(d^{2} N /\left(2 \pi p_{T} d p_{T} d y\right)\right)$ evaluated at the average transverse momentum $\left(p_{T} /|B|=0.875 \mathrm{GeV} / \mathrm{c}\right)$ region versus baryon number distribution. The solid symbols represent our coalescence model calculation, which can fit the measured data points very well. By fitting the model calculation with an exponential function $e^{-r|B|}$, a reduction rate of 1692 (1285) can be obtained 
for each additional antinucleon (nucleon) added to antinucleus (nucleus), compared to $1.6_{-0.6}^{+1.0} \times 10^{3}$ $\left(1.1_{-0.2}^{+0.3} \times 10^{3}\right)$ for nucleus and (antinucleus) obtained by the STAR experiment. The yield of next stable antinucleus (antilithium-6) is predicted to be reduce by a factor of $2.6 \times 10^{6}$ compare to ${ }^{4} \overline{\mathrm{He}}$, and is impossible to be produced within current accelerator technology. The excitation of (anti)nucleus from a highly correlated vacuum was discussed in reference [23]. This new production mechanism can be tested with the measurement of the production rate of (anti)nucleus, any deviation of the production rate of (anti)nucleus from usual reduction rate, may indicate the existence of the direct excitation mechanism. The low production rate of ${ }^{4} \overline{\mathrm{He}}$ antinucleus in nuclear interaction implies that any observation of ${ }^{4} \overline{\mathrm{He}}$ or even heavier antinucleus should be indicative of the existence of a large amount of antimatter somewhere in the Universe.

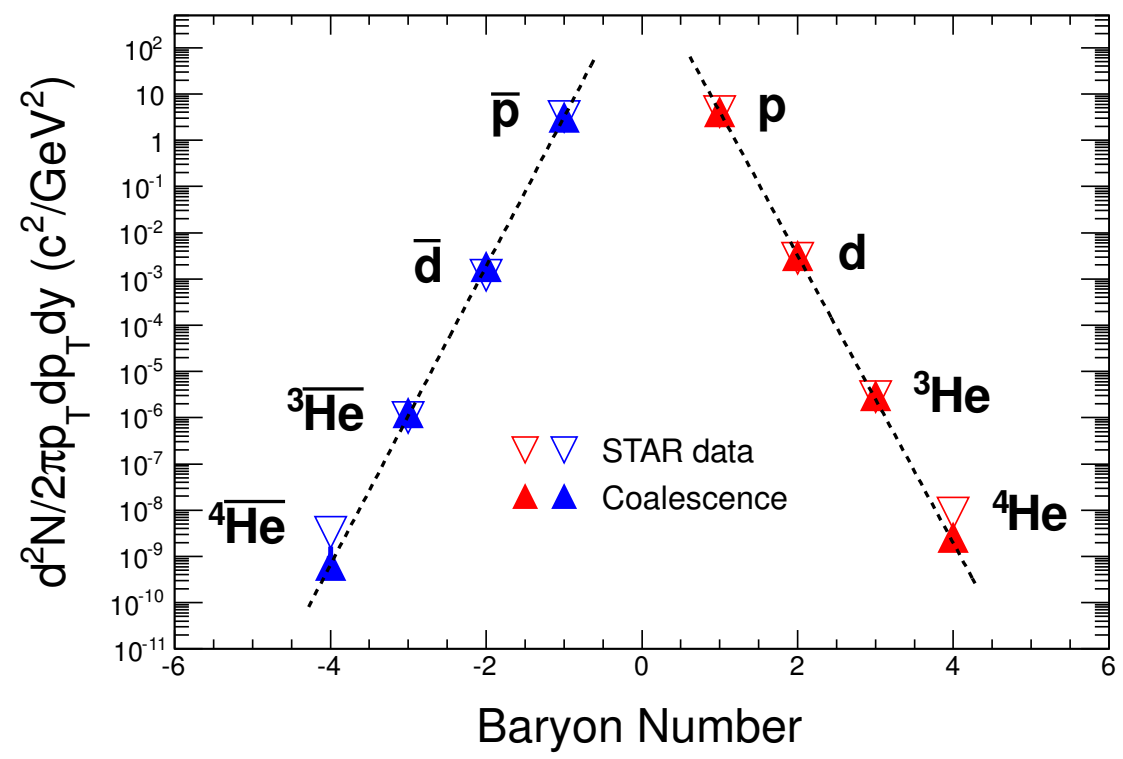

Figure 5. Invariant yields $d^{2} N /\left(2 \pi p_{T} d p_{T} d y\right)$ of (anti)nucleus at the average transverse momentum region $\left(p_{T} /|B|=0.875 \mathrm{GeV} / \mathrm{c}\right)$ as a function of baryon number (B). The open symbols represents the data points extracted by the STAR experiment at RHIC energy, while solid ones are reproduced by coalescence model. The lines represent the exponential fit for our coalescence results of positive particles (right) and negative particles (left) with the formula $e^{-r|B|}$. Adapted from Ref. [21].

\section{Summary}

A brief review is presented for the discovery of ${ }^{4} \overline{\mathrm{He}}$ which is the heaviest antimatter nucleus observed so far [8] and ${ }_{\bar{\Lambda}}^{3} \overline{\mathrm{H}}$ which is the first antimatter hypernucleus [7]. The RHIC has been demonstrated as an excellent facility for antimatter production by the observation of both anti-nuclei. However, with the further increasing of mass of antimatter particles, the difficulty of observation becomes much larger. The searching for some exotic particles is still an on-going programe. To interpret the antimatter nuclei production mechansim, both thermal model and coalescence model can essentially describe the production yield of antimatter and antimatter-matter ratio. A recent calculation based 
on the hydrodynamic motivated BlastWave model coupled with a coalescence model at RHIC energy has demonstrated that the current approach can reproduce the differential invariant yields and relative production abundances of light antinuclei and antihypernuclei [21]. By extrapolating the exponential behavior of the differential invariant yields versus baryon number distribution to $B=-6$ region, the production rate of ${ }^{6} \overline{\mathrm{Li}}$ which is a next stable antinucleus at RHIC is about $10^{-16}$, its observation with the current accelerator technology seems impractical. Of course, any observation of ${ }^{4} \overline{\mathrm{He}}$ in Cosmic rays will be a great hint of the existence of massive antimatter in Universe. Model calculations and experimental measurements in high energy heavy ion collisions can simulate the interactions between high energy protons and interstellar materials. Thus current STAR results and model calculations provide a good background estimation for the future observation of ${ }^{4} \overline{\mathrm{He}}$ and even heavier antinuclei in Universe.

This work is partially supported by the 973 program under contract 2014CB845401, NSFC under contracts No. 11035009, 11220101005, 11275250 and 10905085, the Knowledge Innovation Project of Chinese Academy of Sciences under Grant No. KJCX2-EW-N01.

\section{References}

[1] BRAHMS Collaboration, I. Arsene et al., Nucl. Phys. A 757 , 1 (2005); PHOBOS Collaboration, B. B. Back et al. Nucl. Phys. A 757, 28 (2005); STAR Collaboration, J. Adams et al., Nucl. Phys. A 757102 (2005); PHENIX Collaboration, S. S. Adcox et al., Nucl. Phys. A 757 , 184 (2005)

[2] A. Schuster, Nature 58, 367 (1898)

[3] P. A. M. Dirac, Proc. R. Soc. Lond. A 117, 610 (1928)

[4] C. Y. Chao, Proc. Nat. Acad. Sci. 16, 431 (1930); C. Y. Chao, Phys. Rev. 36, 1519 (1930)

[5] C. D. Anderson, Phys. Rev. 43 , 491 (1933)

[6] Y. G. Ma, J. H. Chen, L. Xue, Front. Phys. 7 , 637 (2012); Y. G. Ma, J. H. Chen, L. Xue, A. H. Tang, Z. B. Xu, Nucl. Phys. News. 23, 10 (2013)

[7] STAR Collaboration, B. I. Abelev et al., Science 328, 58 (2010)

[8] STAR Collaboration, B. I. Abelev et al., Nature 473, 353 (2011)

[9] ALPHA Collaboration, G. B. Andresen et al., Nature Physics 7 , 558 (2011)

[10] H. Kamada, J. Golak, K. Miyagawa, H. Witala, W. Glockle, Phys. Rev. C 57, 1595 (1998)

[11] J. H. Chen, Nucl. Phys. A 835 , 117 (2010); Nucl. Phys. A 830 , 761c (2009)

[12] R. H. Dalitz, G. Rajasekharan, Phys. Lett. 1, 58 (1962)

[13] Y. H. Zhu, Nucl. Phys. A 904-905, 551c (2013); PhD Thesis (2013), Shanghai Institute of Applied Physics, CAS

[14] C. Amsler et al., Phys. Lett. B 667, 1 (2008)

[15] V. Koch, A. Majumder, J. Randrup, Phys. Rev. Lett. 95, 182301 (2005)

[16] S. Zhang et al., Phys. Lett. B 684, 224 (2010)

[17] J. Steinheimer et al., Phys. Lett. B 714, 85 (2012)

[18] L. Xue, J. Phys. G 38 , 124072 (2011); PhD Thesis (2012), Shanghai Institute of Applied Physics, CAS

[19] N. Sharma, J. Phys. G 38 , 124189 (2011)

[20] A. Andronic, P. Braun-Munzinger, J. Stachele, H. Stöcker, Phys. Lett. B 697, 203 (2011)

[21] L. Xue, Y. G. Ma, J. H. Chen, S. Zhang, Phys. Rev. C85, 064912 (2012)

[22] H. Sato and K. Yazaki. et al., Phys. Lett. B 98, 153 (1981)

[23] W. Greiner, Int. J. Mod. Phys. E 5, 1 (1996) 